\title{
BMJ Open Anti-adalimumab antibodies in psoriasis: lack of clinical utility and laboratory evidence
}

\author{
G Lombardi, ${ }^{1}$ S Perego, ${ }^{1}$ V Sansoni, ${ }^{1}$ M Diani, ${ }^{2}$ G Banfi, ${ }^{1,3}$ G Altomare ${ }^{2,4}$
}

To cite: Lombardi G, Perego S, Sansoni V, et al. Anti-adalimumab antibodies in psoriasis: lack of clinical utility and laboratory evidence. BMJ Open 2016;6: e011941. doi:10.1136/ bmjopen-2016-011941

- Prepublication history for this paper is available online. To view these files please visit the journal online (http://dx.doi.org/10.1136/ bmjopen-2016-011941).

Received 16 March 2016 Revised 10 October 2016 Accepted 10 November 2016

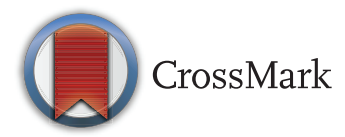

${ }^{1}$ Laboratory of Experimental Biochemistry and Molecular Biology, I.R.C.C.S. Istituto Ortopedico Galeazzi, Milano, Italia

${ }^{2}$ Department of Dermatology and Venereology, I.R.C.C.S. Istituto Ortopedico Galeazzi, Milano, Italia

${ }^{3}$ Vita-Salute San Raffaele University, Milano, Italia ${ }^{4}$ Department of Biomedical Sciences for Health, University of Milano, Milano, Italia

Correspondence to Dr G Lombardi; giovanni. lombardi@grupposandonato. it

\section{ABSTRACT}

Objective: Adalimumab has proven effective in psoriasis; however, secondary failure may result from the drug's immunogenicity. Prevalence data on the immunogenicity of biologicals, and of adalimumab in particular, are highly variable. We investigated the prevalence of anti-adalimumab antibodies and the association with clinical indexes and tumour necrosis factor $\alpha$ (TNF $\alpha$ ) serum levels in psoriatic patients.

Design: Case-control, longitudinal.

Setting: Single centre.

Participants: Patient groups: I $(\mathrm{n}=20)$ receiving biological therapies after switching from adalimumab; II $(\mathrm{n}=30)$ ongoing adalimumab therapy; III $(\mathrm{n}=30)$ novel adalimumab therapy; IV $(n=15)$ biological therapies other than adalimumab.

Healthy subjects: (group $\mathrm{V} ; \mathrm{n}=15$ ) never treated with immunosuppressants or biologicals.

Interventions: All groups were tested at enrolment. Group II was also tested at 12 months, and group III at 1,3 , and 6 months.

\section{Primary and secondary outcome measures:} Standard clinical evaluations (Psoriasis Area Severity Index (PASI)), blood samples and two-site ELISAbased measurement of serum adalimumab trough levels, anti-adalimumab antibodies and TNF $\alpha$.

Results: The false-positive rate was $23 \%$ for adalimumab detection and $22 \%$ for anti-adalimumab antibodies in patients naïve to adalimumab. Spurious positivity for anti-adalimumab antibodies (one-timepoint positivity in group III during follow-up) accounted for $33 \%$ of the total. The prevalence of antidrug antibodies was highest $(87 \%)$ in group I patients. No correlations were found between the presence of anti-adalimumab antibodies or adalimumab levels and changes in PASI scores.

Conclusions: High variability of results, high prevalence of false-positives and lack of association between anti-adalimumab antibodies and TNF $\alpha$ level/ PASI score limit this assay's usefulness. Accurate clinical evaluation is key to early identification of treatment failures.

\section{INTRODUCTION}

Biological therapies have revolutionised the treatment of inflammatory and autoimmune
Strengths and limitations of this study

- The main limitations of this study are: the small number of patients in each group, the correlation analysis between objectively measured parameters (anti-adalimumab antibodies, serum adalimumab trough levels) and a semi-quantitative subjective disease severity index (PASI), and the presence of a subset of patients with psoriatic arthritis.

- The main strength of this study is the exclusion of possible confounding factors that might have affected the analysis.

- Evaluation of four different patient groups: group I previously treated with adalimumab, group II on adalimumab therapy and followed up for 12 months, group III started on adalimumab therapy and followed up for 12 months, and group IV on biological therapies other than adalimumab.

- Exclusion of modifying factors: no cotreatment with immunosuppressants or previous treatments with other biologicals.

- Evaluation of healthy subjects never treated with immunosuppressant drugs and biologicals and psoriatic patients under therapy with biologicals other than adalimumab.

diseases and improved the quality of life of patients over the last decade. By interfering with the activity of key mediators (eg, inflammatory cytokines), biologicals slow down aberrant immune response, thus limiting, or even blocking, the progression of disease in the majority of treated patients. ${ }^{1}$

Psoriasis is a complex disease characterised by interactions between certain genes and environmental factors, leading to an imbalance in immune activation towards the expression of inflammatory mediators known to contribute to the pathogenesis of psoriasis. ${ }^{2}$ Among the mediators successfully targeted by therapeutic agents ${ }^{3}{ }^{4}$ is tumour necrosis factor (TNF). Anti-TNF drugs, initially developed to treat other inflammatory diseases (ie, rheumatoid arthritis), are now used in the treatment of psoriasis and psoriatic arthritis. ${ }^{5}$ Available clinical data indicate 
that response to treatment with anti-TNF agents is effective in $60-70 \%$ of treatment-naïve patients with immunemediated inflammatory diseases. Secondary failure occurs in a certain percentage of cases when ${ }^{6}$ immune reactions against the drug are produced by antibodies targeting the therapeutic molecule (antidrug antibodies -ADA). ${ }^{78}$ Although all protein-based drugs (including anti-TNF agents) are potentially immunogenic, certain structural features of the drug make this event more probable. For instance, the presence of non-human (ie, murine) portions within the molecule increases the probability an immune response will be elicited: the greater the relative weight of non-human sequences, the greater the immunogenic potential of the molecule. By this view, as confirmed by previous studies, chimeric molecules would be generally more immunogenic than humanised and human ones. ${ }^{9} 10$

Anti-TNF agents differ structurally from one another: full-length bivalent IgG antibodies (chimeric: infliximab (IFX), human: adalimumab (ADL), golimumab), polyethylene glycol-conjugated humanised FabI (certolizumab pegol) or human Fc $\gamma 1$-conjugated TNF receptor (TNFR) 2 (etanercept (ETN)). ${ }^{11}$ Among anti-TNF agents, ADL (Humira, Abbvie) has been proven effective in the biological treatment of psoriasis. ${ }^{12-14}$ However, although ADL is a fully human monoclonal antibody against TNF and its immunogenic potential is putatively low, the prevalence data of ADL immunogenicity ranges from $6.4 \%$ to $44.8 \%$ in psoriasis ${ }^{15}$ and from $0.04 \%$ to $87 \%$ in the whole set of ADL-treatable diseases. ${ }^{16}$ Such wide variability, reviewed by several studies, renders this information useless in clinical practice. Moreover, it contrasts with clinical evidence that ADL is highly effective and safe. Indeed, if an acceptable probability of eliciting immune response lies in the lower quartile of this range, in line with clinical observations, it is evident that the upper half of these ranges contemplates a very high probability of immune response, and consequently of secondary therapeutic failure, which is not coherent with reality and, more importantly, would be unacceptable for patients.

There are several possible reasons for this variability. Some have been adequately explained in recent studies (eg, blood sampling at the time of drug trough levels, regular frequency of administration, no change in doses) ${ }^{7}$ whereas other causes have not been fully elucidated. For instance, cotreatment (ie, immunosuppressant drugs-ISs) is variously defined across studies. ISs are known to modify immune response and the response to potential immunogens. ${ }^{17} 18$ This is problematic when patients receiving anti-TNF therapy alone or according to different anti-TNF-ISs cotreatment protocols (simultaneous, alternate, cyclic) are grouped together. Moreover, the potential modifying effects of previous biological therapies are often insufficiently taken into account even though multiple treatments are known to predispose to immunogenicity. ${ }^{15}$

Critical in this context are methodological issues coupled with currently available technologies used to detect/measure ADAs. Different methods have different pitfalls, including low specificity, interference by endogenous compounds and tendency to generate falsepositive results. ${ }^{70}$ As recent studies have failed to fully clarify the clinical relevance of $\mathrm{ADAs},{ }^{19}$ the question remains open whether a real relationship exists between ADA expression and therapeutic failure. Therefore, the aim of this study was to overcome the technical and clinical limitations mentioned above by focusing on ADL treatment for psoriasis in well-characterised groups of ADL-treated patients (formerly treated, under treatment and newly treated), patients treated with other biologicals and healthy controls.

\section{MATERIALS AND METHODS}

\section{Patient population}

Patients with a diagnosis of cutaneous psoriasis and healthy controls were enrolled at the Department of Dermatology and Venereology of I.R.C.C.S. Istituto Ortopedico Galeazzi (Milan, Italia). The study was conducted in accordance with the tenets of the Declaration of Helsinki. Written consent to the use of clinical data in aggregate form was obtained from all patients after being informed about the study procedures.

Patient selection criteria were as follows: absence of comorbidities such as diabetes mellitus, genetic diseases, autoimmune diseases, acute and chronic systemic or cutaneous infections during sample collection, negative results at screening for hepatitis B virus, hepatitis $\mathrm{C}$ virus, HIV and tuberculosis infections. Patients receiving treatment with either IS drugs (cyclosporine A, methotrexate) or systemic corticosteroids during anti-TNF therapy or within 3 weeks before the beginning of the therapy were also excluded from the study.

Administration of ADL therapy followed the standard schedule: $80 \mathrm{mg}$ s.c. in the first infusion, $40 \mathrm{mg}$ after 1 -week, and then $40 \mathrm{mg}$ every 15 days. The clinical severity of psoriasis was scored using the Psoriasis Area Severity Index (PASI) at each visit. ${ }^{20}$

The study sample was composed of four patient groups plus one group of healthy volunteers that served as controls.

- Group I (ex-ADL; $n=20)$ : Previously treated with ADL, which had been discontinued at least 6 months before enrolment in the study, and on treatment with another biological agent (IFX or ETN) at the time of enrolment. Group I was tested once at enrolment.

- Group II (on-ADL; $n=30$ ): Under ADL treatment for at least 6 months prior to the time of enrolment and continued with therapy for at least 12 months after recruitment. Group II was tested twice, at enrolment and at 12 months.

- Group III (naïve; n=30): Never treated with any biological, were started on ADL therapy and continued with therapy for at least 6 months. Group III was tested four times: at enrolment, before the start of therapy (T0), at 1 (T1), 3 (T3) and 6 (T6) months of treatment. 
- Group IV (on-BIO; n=15): Under treatment, or previously treated, for at least 6 months, with biologicals other than ADL (IFX or ETN) and never treated with ADL. Group IV was tested once at enrolment.

- Group V (CTRL; n=15): Healthy controls with no chronic condition and never treated with ISs or biologicals. Group V was tested once at enrolment.

Age at recruitment ranged from 19 to 79 years in the psoriatic patient groups and from 25 to 65 years in the control group. Table 1 presents the study population demographics and clinical features.

\section{Blood sampling}

In the patients receiving biological agents (groups I, II, III, IV), blood samples were collected immediately before drug injection at the time of drug trough level. Non-fasting blood samples were collected in SSTII BD Vacutainers (Becton Dickinson, Franklin Lakes, New Jersey, USA); serum was separated by centrifugation $\left(1200 \mathrm{~g}, 10 \mathrm{~min}\right.$ at $\left.4^{\circ} \mathrm{C}\right)$ and stored at $-80^{\circ} \mathrm{C}$ until assayed. Serum ADL (sADL) trough levels and anti-ADL antibody (sAAA) levels were measured in duplicate using specific two-site (bridging) immunoassays (Promonitor ADL/AAA, Progenika Biopharma, Derio, Spain). Serum TNF $\alpha$ (sTNF $\alpha$ ) was measured using an ultrasensitive ELISA kit (Novex, Life Technologies, Carlsbad, California, USA). The lower limit of detection (LLD) of the assays and the intra-assay variability $\left(\mathrm{CV}_{\mathrm{i}}\right)$ and the interassay variability $\left(\mathrm{CV}_{\mathrm{b}}\right)$ were $3.5 \mathrm{AU} / \mathrm{mL}, 6.6 \%$ and $6.6 \%$ for $\mathrm{sAAA}$, respectively; $0.024 \mu \mathrm{g} / \mathrm{mL}, 6.1 \%$ and $5.1 \%$ for sADL, respectively; and $0.09 \mathrm{pg} / \mathrm{mL}, 6.7 \%$ and $9.7 \%$ for $\mathrm{TNF} \alpha$, respectively. All samples were tested at least twice for confirmation.

Testing for antinuclear antibodies (ANA) and C reactive protein (CRP) was ordered by the patients' physician as needed and according to clinical practice. All tests were performed by accredited laboratories.

\section{Statistical analysis}

Normality of distribution was tested using the D'Agostino-Pearson test. As the values were nonparametrically distributed, quantitative parameters are expressed as the median (range) in the descriptive analysis. Intergroup comparisons were performed using the Kruskal-Wallis test. Spearman's rank correlation test was used to evaluate correlations. Correlations were considered significant when $r \geq 0.25$ for $p<0.05$. Analysis was carried out using GraphPad Prism V.6.01 (GraphPad Software, La Jolla, California, USA). Significance was set at $\mathrm{p}<0.05$.

\section{RESULTS}

\section{Immunogenicity of ADL}

Results are presented in table 2. Detectable sADL levels were found in at least one assay of samples from eight out of 20 ex-ADL patients (group I), but only in one of these eight was the result replicated in the subsequent tests. The variability between the tests, performed on aliquots of the same sample (interassay variability), was very high (interassay mean fold change 50). Notably, the time since discontinuation of ADL therapy ranged from 13 months to 7 years in the patients in which positivity was not confirmed and was only 6 months in the one patient in whom ADL positivity was confirmed. sAAA were detectable in 13 out of 20 ex-ADL patients. Although the interassay variability was high (interassay mean fold change 23), all positive results were replicated.

At recruitment, 26 (87\%) on-ADL patients (group II) had detectable trough ADL concentrations $(5.8 \mu \mathrm{g} / \mathrm{mL}$ (range, 0.1-12.0)). The four patients with undetectable sADL had, however, detectable sAAA, as did two of those with detectable sADL (sAAA $219.3 \mathrm{AU} / \mathrm{mL}$ (range, 141.0-822.2)). At recruitment, all group II patients were classified as responders. There was no significant difference in the median PASI score between the sAAA-negative patients (1.1 (range, 0-14)) and the sAAA-positive patients (4.0 (range, $0.2-8.0$ ) $; \mathrm{p}=0.069$ ) or in the TNF $\alpha$ concentration $(0.12 \mathrm{pg} / \mathrm{mL}$ (range, $0-1.42$ ) vs $0.04 \mathrm{pg} / \mathrm{mL}$ (range, $0-0.54$ ); $\mathrm{p}=0.471$ ) and the CRP concentration $(0.12 \mathrm{mg} / \mathrm{dL}$ (range, $0-8.0)$ vs $0.46 \mathrm{mg} /$ dL (range, 0.13-0.80)), respectively. Furthermore, the percentage of arthropathic patients was $50 \%$ among the patients that tested sAAA-negative and sAAA-positive. After 12 months of ADL therapy, the median change in PASI score was 0.1 in the patients who had tested sAAA-negative at recruitment and 0.2 in those who had tested sAAA-positive at recruitment $(\mathrm{p}=0.673)$. Two patients tested sAAA-positive at 12 months: one with no change in PASI score and one with a decrease to -0.3 . Two patients who had tested sAAA-positive at recruitment had no detectable sAAA at 12 months; the PASI score was unchanged in one patient and increased by 0.8 in one patient. Finally, two patients remained sAAA-positive during the study; the PASI score was decreased by 1 point in one patient and remained unchanged in one patient.

Also, in group II the concordance between replicated tests was low: one patient tested positive for sADL and two patients for sAAA at the first test and negative at the three subsequent tests. The mean fold change between replicated tests was 34 for sADL and 26 for sAAA.

Among the subjects never exposed to ADL, 14 tested sADL-positive (three from group III (naïve) at T0, six from group IV (on-BIO) and five from group V (CTRL)) and 13 tested sAAA-positive (one from group III at T0, seven from group IV and five from group V). The positive samples were detected in one or more of at least two replicated assays. The overall frank falsepositivity rate was $23 \%$ for sADL and $22 \%$ for sAAA.

During the 6-month follow-up, 13 out of 30 (43\%) group III patients had detectable sAAA levels at one or more time points after T0. Notably, in all these cases but three, sAAA expression was spurious because it was found at a single time point (at either 1 or 3 months). 
Table 1 Characteristics of the study population at recruitment

\begin{tabular}{|c|c|c|c|c|c|c|}
\hline & \multicolumn{5}{|l|}{ Cases } & \multirow{2}{*}{$\begin{array}{l}\text { Controls } \\
\text { Group V CTRL, } \\
n=15\end{array}$} \\
\hline & $\begin{array}{l}\text { Total patients, } \\
\mathrm{n}=95\end{array}$ & $\begin{array}{l}\text { Group I ex-ADL, } \\
\mathrm{n}=20\end{array}$ & $\begin{array}{l}\text { Group II on-ADL, } \\
\mathrm{n}=30\end{array}$ & $\begin{array}{l}\text { Group III naïve, } \\
\mathrm{n}=30\end{array}$ & $\begin{array}{l}\text { Group IV on-BIO, } \\
n=15\end{array}$ & \\
\hline Age, median (range) years & $48.0(19.0-79.0)$ & $49.5(28.0-79.0)$ & $48.0(21.0-65.0)$ & $48.5(19.0-66.0)$ & $47.0(29.0-70.0)$ & $34(25-65)$ \\
\hline Male, n (\%) & $71(74.7)$ & $14(70.0)$ & 28 (93.3) & $24(80.0)$ & $5(33.3)$ & 7 (46.7) \\
\hline Female, $\mathrm{n}(\%)$ & $24(25.3)$ & $6(30.0)$ & $2(6.7)$ & $6(20.0)$ & $10(76.7)$ & $8(53.3)$ \\
\hline BMI, median (range) $\mathrm{kg} / \mathrm{m}^{2}$ & $26.8(18.0-44.0)$ & $28.0(20.0-39.4)$ & $26.5(20.5-32.8)$ & $16.2(20.0-44.0)$ & $26.5(18.0-39.4)$ & $23(19.0-27.0)$ \\
\hline $\begin{array}{l}\text { Disease duration, median } \\
\text { (range) years }\end{array}$ & $20.0(4.0-60.0)$ & $16.0(7.0-56.0)$ & $20.0(4.0-60.0)$ & $18.5(4.0-60.0)$ & $32.0(10.0-56.0)$ & - \\
\hline PASI, median (range) & $3.0(0.0-24.0)$ & $2.0(0.0-24.0)$ & $1.1(0.0-14.0)$ & $12.2(2.5-22.0)$ & $0.0(0.0-14.0)$ & - \\
\hline PsA, n (\%) & $45(47.4)$ & $10(50.0)$ & $15(50.0)$ & $11(36.6)$ & $9(60.0)$ & - \\
\hline Previous treatments, $\mathrm{n}(\%)$ & $95(100)$ & $20(100)$ & $30(100)$ & $30(100)$ & $15(100)$ & - \\
\hline Retinoids & $8(8.4)$ & $3(15.0)$ & $2(6.7)$ & 1 (3.3) & 2 (13.3) & - \\
\hline UV B & $19(20.0)$ & $5(25.0)$ & $6(20.0)$ & $8(26.7)$ & $0(0)$ & - \\
\hline MTX & $76(80.0)$ & $13(65.0)$ & 25 (83.3) & 26 (86.7) & $12(80.0)$ & - \\
\hline CsA & 62 (65.3) & $12(60.0)$ & $21(70.0)$ & $21(70.0)$ & 8 (53.3) & - \\
\hline ETN & $26(27.4)$ & $11(55.0)$ & $9(30.0)$ & $0(0)$ & $6(40.0)$ & - \\
\hline IFX & $15(15.8)$ & $1(5.0)$ & $2(6.7)$ & $0(0)$ & $12(80.0)$ & - \\
\hline EFZ & 7 (7.4) & $2(10.0)$ & $2(6.7)$ & $0(0)$ & $3(20.0)$ & - \\
\hline ADL & $20(21.0)$ & $20(100)$ & $0(0)$ & $0(0)$ & $0(0)$ & - \\
\hline USK & $5(5.3)$ & $3(15.0)$ & $0(0)$ & $0(0)$ & 2 (13.3) & - \\
\hline GOL & $1(1.0)$ & $0(0)$ & $1(3.3)$ & $0(0)$ & $0(0)$ & - \\
\hline
\end{tabular}

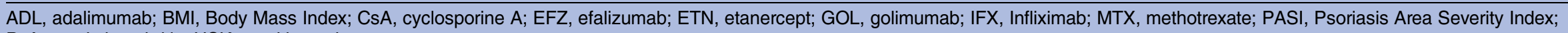
PsA, psoriatic arthritis; USK, ustekinumab. 


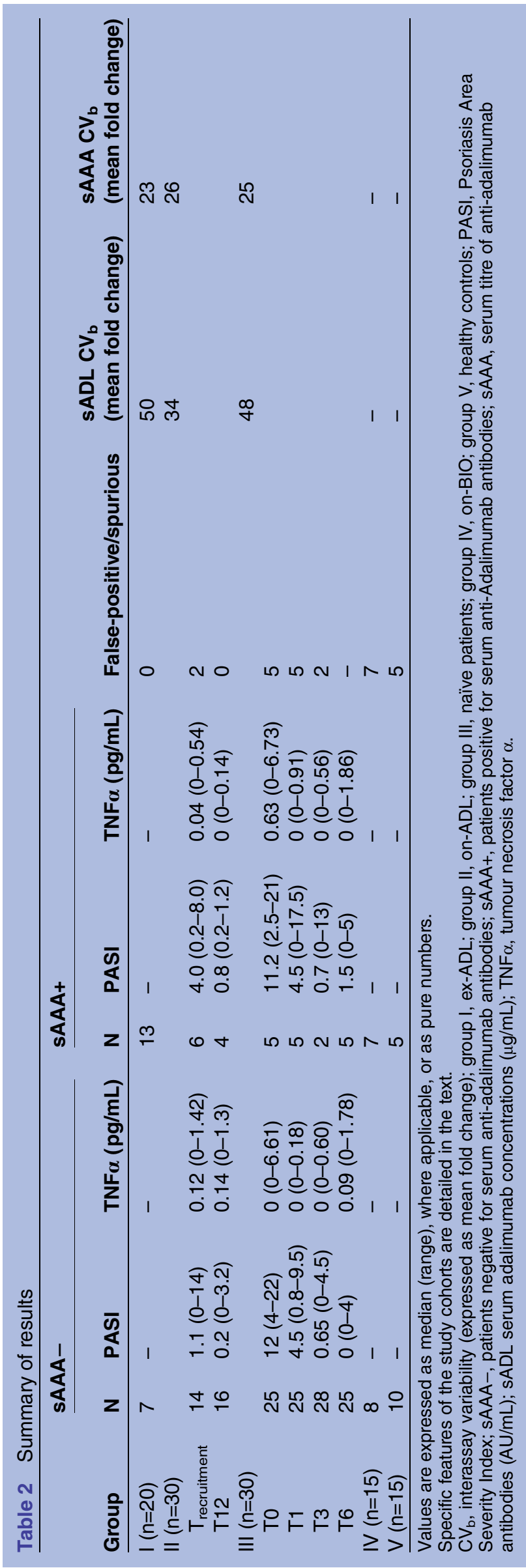

Only five patients $(16.7 \%)$ tested sAAA-positive at 6 months, which could have been due to a real immunogenic event. The median change in PASI scores between the patients testing sAAA-negative or sAAA-positive (at least once) was -12.0 (range, -4.0 to -22.0 ) and -9.3 (range, 0.0 to -21.0 ), respectively, without any significant difference $(\mathrm{p}=0.105)$. No significant difference in PASI scores was observed when the patients with no sAAA and those spuriously expressing sAAA were compared with those with detectable sAAA only at 6 months $(-12.0$ (range, -2.0 to -22.0 ) versus -9.3 (range, 0.0 to $-15.1) ; \mathrm{p}=0.273)$. The variability between replicates was very high, with an interassay mean fold change of 48 for sADL and 25 for sAAA, as was the intra-assay variability (concordance between duplicates), adjusted for the relative dilution factor, which ranged from $10 \%$ to $83 \%$ for both tests.

\section{Correlation between immunogenicity and humoral and clinical parameters}

There was a statistically significant correlation between positivity for SAAA (but not the titre) and TNFa levels in group I patients $(\mathrm{r}=0.56 ; \mathrm{p}=0.025)$. In group II, there was a clinically significant correlation between CRP concentration and duration of disease $(\mathrm{r}=0.54 ; \mathrm{p}=0.032)$ and an inverse correlation between the presence of psoriatic arthritis and age $(\mathrm{r}=-0.42 ; \mathrm{p}=0.015)$. sAAA positivity and concentrations were inversely correlated with age $(\mathrm{r}=$ $-0.37 ; \mathrm{p}=0.036)$ and sADL $(\mathrm{r}=-0.52 ; \mathrm{p}=0.002)$. The PASI score was inversely correlated with sAAA concentration $(\mathrm{r}=-0.38 ; \mathrm{p}=0.033)$ and with $\mathrm{sADL}$ concentration $(\mathrm{r}=$ $-0.65 ; \mathrm{p}<0.001)$. In group III, sAAA positivity was inversely correlated with detectable levels of sADL ( $\mathrm{r}=$ $-0.27 ; \mathrm{p}=0.003)$. Neither sAAA nor sADL concentration was correlated with the presence of psoriatic arthritis, presence of ANA or circulating CRP concentrations.

\section{DISCUSSION}

The main finding of this study is the low reliability of these assay kits in determining AAA expression and sADL trough levels. Multiple factors can contribute to low assay reliability. Some are merely methodological: the high number of false-positive results, as reported elsewhere, ${ }^{21} 22$ and the low concordance of the results after retesting in the current study indicating low specificity and possible external transitory interferences. Others are clinical: a generally low occurrence of AAA detected in this population of patients $(16.7 \%)$, except for frankly false-positive results, in addition to a low concordance between AAA positivity and the clinical course of the disease, as reflected by the changes in PASI scores and the TNF $\alpha$ and CRP levels. A different situation emerged when immunogenicity was detected in patients previously exposed to ADL whose therapy was switched to another biological due to secondary failure. In this case, the prevalence of sAAA was $87 \%$. 
Previous studies have investigated the expression of ADAs in patients treated with different biological agents for inflammatory and autoimmune diseases. Although many were well-designed studies involving large, wellcharacterised cohorts, the general experimental settings were essentially the same: either one-time evaluation of patients receiving the drug or longitudinal evaluation of patients at the first treatment and then again at 6 or 12 months. ${ }^{23}$ While the pioneering studies on the immunogenicity of biologicals did not take into account timing of sampling, later research recognised this as an important aspect and used samples taken just before drug administration when circulating drug concentrations are lowest. This holds particular importance because such assays are inhibited by the presence of the drug in serum. ${ }^{22} 24$ Although the low specificity of these assays has been repeatedly reported, ${ }^{21}$ to the best of our knowledge, no studies to date have addressed this issue in practice. Here, we aimed to overcome the principal limitations of previous studies in order to obtain a clearer picture of immune response to biologicals, and of immunogenicity of the anti-TNF agent ADL in particular.

To do this, we employed two complementary strategies: sample retesting (for both ADL and AAA) in order to confirm (or reject) the test result and inclusion of subjects (healthy and psoriatic) never treated with ADL as negative controls. The second strategy sets out study apart from previous studies in which the cohorts often included patients treated with biologicals and patients who, due to clinical needs, received concurrent treatment with biologicals and ISs, which, however, are known to modify the overall immunogenic response. ${ }^{18} 25$ Furthermore, we used a two-site (bridging) assay which, along with radioimmunoassay (RIA) and antigen binding test (ABT), is considered the most reliable test in this specific setting. ${ }^{26}$ The two-site assay is based on the ability of antidrug IgGs (types 1, 2 and 3) to crosslink the antibody molecule of the drug coated onto the plate and the labelled antibody drug added to the reaction solution. Although this assay is specific and sensitive, it does not detect IgG4 due to their bi-specificity. ${ }^{27}$ In addition, we measured serum TNF levels. Our rationale was that, because TNF is the target of the anti-TNF agent, and ADAs are, as claimed, ${ }^{28}$ neutralising against the drug, TNF levels would be expected to rise. Therefore, it appeared appropriate to measure TNF concentrations to test the neutralising potential of eventual ADAs.

The main critical issues of this study are the small sample size and the choice of the ELISA method to detect sAAA. To address the former, we excluded patients receiving concurrent IS-biological therapies, which is a common practice in the treatment of psoriasis. In this way, we were able to select for cohorts that were highly homogeneous, except for the subset of patients with psoriatic arthritis. From a purely mathematical point of view, the statistical reliability was reduced due to the low median PASI score, at least in group II, and the small sample size, as was the correlation analysis between an objective index (AAA) and a semiquantitative, well-validated but subjective index (PASI). It is opinionable whether an objective index of disease severity can be correlated with the putative index of treatment analysed here. The second issue concerns the choice of a standard ELISA method. This was dictated by the need to use a method that is more likely to be implemented in routine settings than RIA (due to radioactivity) or in-house modifications of the ELISA protocol (eg, pH-shift-anti-idiotype ABT, ${ }^{29}$ mobility-shift assay ${ }^{30}$ ).

Although the molecular structure of ADL is completely of human origin, it can elicit an immune response in some cases. The prevalence data on anti-ADL immunogenicity vary widely $(0.04-87 \%),{ }^{16}$ however, rendering them clinically meaningless. When interpreting these rates, it is important to note that the study reporting the lower rate $(0.04 \%)$ lasted only 4 weeks, ${ }^{31}$ which is a too short a time for immunogenicity to develop, ${ }^{32}$ while the one reporting the upper rate, although it lasted 45 weeks, involved only 15 subjects. ${ }^{33}$ Our data indicate that the immunogenicity potential of ADL locates in the lower half of the range.

Our data substantiate previous reports of the prevalence of AAA. Despite the homogeneity of the cohorts in our study, the exclusion of potential modifying factors (ie, no IS cotreatment, same treatment doses and protocol) and testing at the time of sADL trough levels, we found a wide quantitative variability in the laboratory data. Moreover, the prevalence of AAA in our sample was low. Finally, neither the serum TNF $\alpha$ concentration nor the PASI score was associated with AAA expression in the patients receiving ADL therapy.

What should be kept in mind is that immunogenicity is a gradual process that develops and changes over time. Continuation of treatment may induce either tolerance or stimulate further antibody production. Also, it has been reported that antibodies against IFX or ADL become undetectable in some patients on continuation of treatment or dose escalation. ${ }^{34} 35$

The methodological considerations mentioned above, and confirmed here, along with the increasing number of commercially available assays, explain the high variability of prevalence data, but they also considerably limit the credibility of the data from clinical studies in which ADAs are evaluated with assays developed by drug companies, without providing complete information about the technology used.

In conclusion, future research will need to investigate the immunogenicity of biologicals according to protocols that can provide a measurement of the reliability of the assay used. For the time being, the reasons remain to be elucidated for this low specificity (ie, the high number of false-positive results) and the substances/situations possibly interfering with ADL/AAA measurement.

Acknowledgements The authors are indebted to Drs Elena Frigerio, Chiara Franchi, Cecilia Garutti, Samantha Spadino, Andrea Altomare, Matteo

C. Garavaglia, Vincenzo Grasso, Professor Paolo Pigatto, Lucrezia Bertino and Chiara Cozzi for their invaluable support in patient recruitment. 
Contributors GL was involved in study design, study coordination, data analysis and manuscript writing. SP and VS contributed to biochemical testing and data analysis. MD was involved in patient recruitment coordination and manuscript writing. GB was involved in study design and critical revision of the manuscript. GA contributed to study design, study coordination and critical revision of the manuscript.

\section{Funding The Italian Ministry of Health (Ministero della Salute).}

Competing interests All authors have completed the ICMJE uniform disclosure form at http://www.icmje.org/coi_disclosure.pdf and declare no support from any organisation for the submitted work; SP, VS, MD, GB have no competing interests. GL has been a speaker for Eli Lilly. GA has been a speaker for MSD, AbbVie, Novartis, Leo Pharma and Eli Lilly. There are no other relationships or activities that could appear to have influenced the submitted work.

\section{Patient consent Obtained.}

Ethics approval Institutional Review Board of I.R.C.C.S. Istituto Ortopedico Galeazzi (Milan, Italia).

Provenance and peer review Not commissioned; externally peer reviewed.

Data sharing statement No additional data are available.

Open Access This is an Open Access article distributed in accordance with the Creative Commons Attribution Non Commercial (CC BY-NC 4.0) license, which permits others to distribute, remix, adapt, build upon this work noncommercially, and license their derivative works on different terms, provided the original work is properly cited and the use is non-commercial. See: http:// creativecommons.org/licenses/by-nc/4.0/

\section{REFERENCES}

1. Lambert J, Nast A, Nestle FO, et al. Practical guidance on immunogenicity to biologic agents used in the treatment of psoriasis: What can be learnt from other diseases? J Dermatol Treat 2015;26:520-7

2. Arican $\mathrm{O}$, Aral M, Sasmaz $\mathrm{S}$, et al. Serum levels of TNF-alpha, IFN-gamma, IL-6, IL-8, IL-12, IL-17, and IL-18 in patients with active psoriasis and correlation with disease severity. Med Inflamm 2005;2005:273-9.

3. Harden JL, Krueger JG, Bowcock AM. The immunogenetics of psoriasis: a comprehensive review. J Autoimmun 2015;64:66-73.

4. Prieto-Pérez R, Cabaleiro T, Daudén E, et al. Genetics of psoriasis and pharmacogenetics of biological drugs. Autoimmune Dis 2013;2013:613086.

5. Sandoval LF, Pierce A, Feldman SR. Systemic therapies for psoriasis: an evidence-based update. Am J Clin Dermatol 2014:15:165-80.

6. Gniadecki R, Bang B, Bryld LE, et al. Comparison of long-term drug survival and safety of biologic agents in patients with psoriasis vulgaris. Br J Dermatol 2015;172:244-52.

7. Vincent FB, Morand EF, Murphy K, et al. Antidrug antibodies (ADAb) to tumour necrosis factor (TNF)-specific neutralising agents in chronic inflammatory diseases: a real issue, a clinical perspective. Ann Rheum Dis 2013;72:165-78.

8. Chaparro M, Guerra I, Muñoz-Linares $P$, et al. Systematic review: antibodies and anti-TNF-alpha levels in inflammatory bowel disease. Aliment Pharmacol Ther 2012;35:971-86.

9. De Groot AS, Terry F, Cousens L, et al. Beyond humanization and de-immunization: tolerization as a method for reducing the immunogenicity of biologics. Expert Rev Clin Pharmacol 2013:6:651-62.

10. Emi Aikawa N, de Carvalho JF, Artur Almeida Silva C, et al. Immunogenicity of Anti-TNF-alpha agents in autoimmune diseases. Clin Rev Allergy Immunol 2010;38:82-9.

11. Tracey D, Klareskog L, Sasso EH, et al. Tumor necrosis factor antagonist mechanisms of action: a comprehensive review. Pharmacol Ther 2008;117:244-79.

12. Menter A, Tyring SK, Gordon K, et al. Adalimumab therapy for moderate to severe psoriasis: a randomized, controlled phase III trial. J Am Acad Dermatol 2008;58:106-15.

13. Asahina $A$, Nakagawa $H$, Etoh $T$, et al. Adalimumab in Japanese patients with moderate to severe chronic plaque psoriasis: efficacy and safety results from a Phase II/III randomized controlled study. J Dermatol 2010;37:299-310.

14. Papp K, Crowley J, Ortonne JP, et al. Adalimumab for moderate to severe chronic plaque psoriasis: efficacy and safety of retreatment and disease recurrence following withdrawal from therapy. Br J Dermatol 2011;164:434-41.

15. Hsu L, Armstrong AW. Anti-drug antibodies in psoriasis: a critical evaluation of clinical significance and impact on treatment response. Expert Rev Clin Immunol 2013;9:949-58.

16. De Simone C, Amerio P, Amoruso G, et al. Immunogenicity of anti-TNF $\alpha$ therapy in psoriasis: a clinical issue? Expert Opin Biol Ther 2013;13:1673-82.

17. Vermeire S, Noman M, Van Assche G, et al. Effectiveness of concomitant immunosuppressive therapy in suppressing the formation of antibodies to infliximab in Crohn's disease. Gut 2007;56:1226-31.

18. Radstake TR, Svenson M, Eijsbouts AM, et al. Formation of antibodies against infliximab and adalimumab strongly correlates with functional drug levels and clinical responses in rheumatoid arthritis. Ann Rheum Dis 2009;68:1739-45.

19. Callis Duffin K. Preventing and detecting antibodies to anti-tumor necrosis factor agents: gaps in practice or in evidence? JAMA Dermatol 2014:150:136-7.

20. Menter A, Gottlieb A, Feldman SR, et al. Guidelines of care for the management of psoriasis and psoriatic arthritis: Section 1. Overview of psoriasis and guidelines of care for the treatment of psoriasis with biologics. J Am Acad Dermatol 2008;58:826-50.

21. Hart $\mathrm{MH}$, de Vrieze $\mathrm{H}$, Wouters $\mathrm{D}$, et al. Differential effect of drug interference in immunogenicity assays. J Immunol Methods 2011;372:196-203.

22. Jamnitski A, Krieckaert CL, Nurmohamed MT, et al. Patients non-responding to etanercept obtain lower etanercept concentrations compared with responding patients. Ann Rheum Dis 2012;71:88-91.

23. Garcês S, Demengeot J, Benito-Garcia E. The immunogenicity of anti-TNF therapy in immune-mediated inflammatory diseases: a systematic review of the literature with a meta-analysis. Ann Rheum Dis 2013;72:1947-55

24. Wolbink GJ, Aarden LA, Dijkmans BA. Dealing with immunogenicity of biologicals: assessment and clinical relevance. Curr Opin Rheumatol 2009;21:211-15.

25. Jani M, Barton A, Warren RB, et al. The role of DMARDs in reducing the immunogenicity of TNF inhibitors in chronic inflammatory diseases. Rheumatol 2014;53:213-22.

26. Aarden L, Ruuls SR, Wolbink G. Immunogenicity of anti-tumor necrosis factor antibodies-toward improved methods of anti-antibody measurement. Curr Opin Immunol 2008;20:431-5.

27. van der Neut Kolfschoten M, Schuurman J, Losen M, et al. Anti-inflammatory activity of human IgG4 antibodies by dynamic Fab arm exchange. Science 2007;317:1554-7.

28. van Schouwenburg PA, van de Stadt LA, de Jong RN, et al. Adalimumab elicits a restricted anti-idiotypic antibody response in autoimmune patients resulting in functional neutralisation. Ann Rheum Dis 2013;72:104-9.

29. van Schouwenburg PA, Krieckaert CL, Rispens T, et al. Long-term measurement of anti-adalimumab using $\mathrm{pH}$-shift-anti-idiotype antigen binding test shows predictive value and transient antibody formation. Ann Rheum Dis 2013;72:1680-6.

30. Wang SL, Hauenstein S, Ohrmund L, et al. Monitoring of adalimumab and antibodies-to-adalimumab levels in patient serum by the homogeneous mobility shift assay. J Pharm Biomed Anal 2013;78-79:39-44.

31. Hanauer SB, Sandborn WJ, Rutgeerts $P$, et al. Human anti-tumor necrosis factor monoclonal antibody (adalimumab) in Crohn's disease: the CLASSIC-I trial. Gastroenterology 2006;130:323-33; quiz 591.

32. Bartelds GM, Krieckaert CL, Nurmohamed MT, et al. Development of antidrug antibodies against adalimumab and association with disease activity and treatment failure during long-term follow-up. JAMA 2011;305:1460-8.

33. Bender NK, Heilig CE, Droll B, et al. Immunogenicity, efficacy and adverse events of adalimumab in RA patients. Rheumatol Int 2007;27:269-74.

34. Wolbink GJ, Vis M, Lems W, et al. Development of antiinfliximab antibodies and relationship to clinical response in patients with rheumatoid arthritis. Arthritis Rheum 2006;54:711-15

35. Bartelds GM, Wijbrandts CA, Nurmohamed MT, et al. Clinical response to adalimumab: relationship to anti-adalimumab antibodies and serum adalimumab concentrations in rheumatoid arthritis. Ann Rheum Dis 2007;66:921-6. 